Pacific Journal of Mathematics

DISINTEGRATION OF KMS-STATES AND REDUCTION OF 


\title{
DISINTEGRATION OF KMS-STATES AND REDUCTION OF STANDARD VON NEUMANN ALGEBRAS
}

\author{
NORBERT RIEDEL
}

\begin{abstract}
It is shown that any von Neumann algebra $\mathscr{N}$ with a cyclic and separating vector can be decomposed into factors in such a manner that the type of $\Re$ is preserved under this decomposition.
\end{abstract}

Introduction. Since the classical work of von Neumann [9] on reduction theory appeared in 1949, several attempts have been undertaken by other authors to generalize the results of von Neumann to operator algebras which are not necessarily countably generated. We want to mention only a few more recent papers, namely those of Halpern $[4,5]$, Strătilă and Zsidó [18, 19], and Teleman [29, 30]. In Halpern [4] as well as in Strătilă and Zsidó [18], it was shown in a similar way that every von Neumann algebra of type I and type II can be decomposed into factors of type I and II, respectively. In Teleman [29, 30] Choquet theory was applied in order to obtain a decomposition into factors of an arbitrary von Neumann algebra.

In the present paper we shall use the theory of standard von Neumann algebras, as well as Choquet theory, in order to show that any von Neumann algebra $\Re$ with a cyclic and separating vector can be decomposed into factors in such a manner that the factors which occur in the decomposition preserve the type of $\mathfrak{K}$. For a semifinite von Neumann algebra we shall prove a stronger result, namely there exists a disintegration of the traces which are defined on the positive cone $\Re^{+}$of $\Re$.

It has already been shown by the work of Jurzak $[6,7]$ and Lance $[8]$ and Sutherland $[\mathbf{2 1}, \mathbf{2 2}]$ that even in the "separable" reduction theory the Tomita-Takesaki theory can be used with considerable success to improve the earlier results in this subject. The first author who introduced the methods of Tomita-Takesaki theory into the reduction of general von Neumann algebras seems to be Halpern in his recent paper [5]. Also quite recently the present author has used the methods of Hilbert algebras in $[13,14]$, as well as the methods of left Hilbert algebras, in $[15,16]$ in order to develop a theory of direct integrals which does not depend on the countability axioms which occur in the theory of von Neumann. 
Our method of decomposing von Neumann algebras into factors which we want to present in the sequel is rather transparent. Let $\Re$ be a von Neumann algebra which acts on the Hilbert space $\mathcal{H}$ with a cyclic and separating vector $e$. In particular $\mathfrak{T}$ is standard. Let $\left\{\bar{\sigma}_{t}\right\}_{t \in \mathbf{R}}$ be the corresponding modular automorphism group. We shall show that there exists a $C^{*}$-dynamical system $(\mathbb{Q}, \mathbf{R}, \sigma)$ such that $\mathscr{Q}$ is a weakly dense $C^{*}$-subalgebra of $\mathfrak{T}$, and $\sigma_{t}$ is the restriction of $\bar{\sigma}_{t}$ on $\mathscr{Q}$ for each $t \in \mathbf{R}$. Thus the vector state $\psi$ on $Q$ which is associated with $e$ is a KMS-state and the GNS-representation of $Q$ associated with $\psi$ can be identified with the identical representation of $\mathbb{Q}$. The set $K(\mathbb{Q}, \sigma)$ of all KMS-states on $\mathbb{Q}$ is a Choquet simplex, and a KMS-state $\phi \in K(\mathbb{Q}, \sigma)$ belongs to the set $\partial K(\mathbb{Q}, \sigma)$ of extreme points in $K(\mathbb{Q}, \sigma)$ if and only if the corresponding GNS-representation $\pi_{\phi}$ of $\mathcal{Q}$ is factorial, i.e., the double commutant $\pi_{\phi}(\mathbb{Q})^{\prime \prime}$ of $\pi_{\phi}(\mathbb{Q})$ is a factor. Now we consider the central measure $\mu$ of $\psi$, which is known to be pseudo-concentrated on the set $\partial K(\mathscr{Q}, \sigma)$. Hence we can define a probability measure on $\partial K(\mathbb{Q}, \sigma)$ such that $\mathcal{H}$ is the direct integral of some Hilbert spaces $\mathcal{H}_{\phi}, \psi \in \partial K(\mathcal{Q}, \sigma)$, in the sense of Wils [32]. Moreover, for the operators in $\Re$ there exist (essentially) unique decompositions into operators which are contained in the von Neumann algebras $\pi_{\psi}(\mathscr{Q})^{\prime \prime}$ such that the algebraic operations in $\mathfrak{K}$ are preserved. This yields the desired decomposition of $\mathfrak{N}$ into factors.

The method we have just described suggests considering arbitrary KMS-states on $C^{*}$-algebras also. In fact, the whole procedure goes through in the general situation. Therefore we shall develop our theory as far as possible in the framework of general KMS-states on $C^{*}$-algebras. As a result we obtain, for instance, that for a KMS-state $\psi$ of type III the corresponding central measure $\mu$ is pseudo-concentrated on extremal KMS-states of type III.

The paper is divided into three sections. In $\$ 1$ we study the direct integrals of some fields of left Hilbert algebras. The idea behind this is to give a unified treatment of the decompositions which occur in $\$ 2$ where we investigate the disintegration of general KMS-states. In $\$ 3$ we apply the theory of $\S \S 1$ and 2 to semifinite and type III von Neumann algebras with a cyclic and separating vector.

For the definitions and notations associated with von Neumann algebras, we refer to the books of Strătilă and Zsidó and Takesaki. For the definitions and notations associated with $C^{*}$-algebras, we refer to Pedersen's book. Observe that the definition of crossed product which we use in the sequel is different from Pedersen [10]. We shall quote from Pedersen's book without emphasizing explicitly the necessary notational alterations. 
The author is very grateful to László Zsidó for useful discussions on this work. The idea for the proof of Proposition 3.3 is due to him.

1. Integrable fields of left Hilbert algebras. Let $(\Omega, \Sigma, \nu)$ be a finite measure space. Let $\left\{\mathfrak{A}_{\xi}\right\}_{\xi \in \Omega}$ be a field of left Hilbert algebras. For every $\xi \in \Omega$ we denote by $\mathcal{H}_{\xi}$ the completion of $\mathfrak{A}_{\xi}$. For some subset $\Lambda \subseteq$ $\Pi_{\xi \in \Omega} \mathfrak{A}_{\xi}$ we consider the following conditions:

(1.1) $\Lambda$ is an involutive subalgebra of $\prod_{\xi \in \Omega} \mathfrak{A}_{\xi}$, where the operations in $\Pi_{\xi \in \Omega} \mathfrak{A}_{\xi}$ are defined pointwise; moreover, $\{x(\xi) / x \in \Lambda\}^{\prime \prime}=\mathfrak{A}_{\xi}$ holds for every $\xi \in \Omega$.

(1.2) For any $x \in \Lambda$ the function $\xi \mapsto\|x(\xi)\|$ is square integrable. By (1.2) we can associate with $\Lambda$ an integrable field of Hilbert spaces $\left(\left\{\mathcal{H}_{\xi}\right\}_{\xi \in \Omega}, \hat{\Lambda}\right)$ in the sense of Wils [32], 2.3. Let $\mathcal{H}=\int^{\oplus} \mathcal{H}_{\xi} d \nu(\xi)$ be the corresponding direct integral. For each $x \in \hat{\Lambda}$ we denote by $\tilde{x}$ or $\int^{\oplus} x(\xi) d \nu(\xi)$ the canonical image of $x$ in $\mathcal{H}$. The set $\mathfrak{A}=\{\tilde{x} / x \in \Lambda\}$ is seen to be an algebra with involution. In case $\Lambda$ satisfies axioms (1.1) and (1.2), we consider the following additional conditions:

(1.3) $\mathfrak{A}$ is a left Hilbert algebra which is dense in $\mathcal{H}$, and for every $x \in \Lambda$ the inequality $\|\pi(x(\xi))\| \leq\|\pi(\tilde{x})\|$ holds for every $\xi \in \Omega$. $(\pi$ denotes the left regular representation of the corresponding left Hilbert algebra.)

(1.4) The canonical conjugation $J$ associated with $\mathfrak{A}$ is decomposable and $\xi \mapsto J_{\xi}$ is a decomposition of $J$ (i.e. $J \tilde{x}=\int^{\oplus} J_{\xi} x(\xi) d \nu(\xi)$ holds for each $x \in \hat{\Lambda}), J_{\xi}$ being the canonical conjugation associated with $\mathfrak{A}_{\xi}$ for any $\xi \in \Omega$.

(1.5) There is a sequence $\left\{x_{n}\right\}_{n \in \mathbf{N}}$ in $\hat{\Lambda}$ such that the set $\left\{T x_{n}(\xi) / T\right.$ $\left.\in \mathcal{L}\left(\mathfrak{A}_{\xi}\right)^{\prime}, n \in \mathbf{N}\right\}$ is total in $\mathcal{F}_{\xi}$ for any $\xi \in \Omega$ and the set $\left\{\sigma \tilde{x}_{n} / \sigma \in\right.$ $\left.\mathcal{L}(\mathfrak{U})^{\prime}, n \in \mathbf{N}\right\}$ is total in $\mathcal{H}$.

1.1 Definition. $\left(\left\{\mathfrak{U}_{\xi}\right\}_{\xi \in \Omega}, \Lambda\right)$ is called an integrable field of left Hilbert algebras if $\Lambda$ satisfies (1.1)-(1.5).

For the remainder of this section we assume $\left(\left\{\mathfrak{A}_{\xi}\right\}_{\xi \in \Omega}, \Lambda\right)$ is a fixed integrable field of left Hilbert algebras. Moreover, we assume $\left\{x_{n}\right\}_{n \in \mathbf{N}}$ to be a sequence satisfying (1.5).

1.2 Definition. An operator field $\xi \mapsto T(\xi)$ is called a natural decomposition of some $\mathcal{T} \in \mathfrak{L}(\mathfrak{A})$ if $\xi \mapsto T(\xi)$ is a decomposition of $\mathcal{T}$ and $T(\xi) \in \mathcal{L}\left(\mathfrak{A}_{\xi}\right)$ holds a.e. We shall write $\mathcal{T}=\int^{\oplus} T(\xi) d \nu(\xi)$ in this case. (We shall use this notation also if $T(\xi)$ is defined a.e. only.) 
1.3 Proposition. (a) If $\mathcal{T}=\int^{\oplus} T_{1}(\xi) d \nu(\xi)=\int^{\oplus} T_{2}(\xi) d \nu(\xi)$ holds for some operator $\mathcal{T} \in \mathcal{L}(\mathfrak{A})$, then we have $T_{1}(\xi)=T_{2}(\xi)$ a.e.

(b) If $\mathscr{T}_{1}=\int^{\oplus} T_{1}(\xi) d \nu(\xi), \mathscr{T}_{2}=\int^{\oplus} T_{2}(\xi) d \nu(\xi)$ for two operators $\mathscr{T}_{1}$, $\sigma_{2} \in \mathcal{L}(\mathfrak{U})$, then we have $\lambda \widetilde{T}_{1}+\mu \widetilde{T}_{2}=\int^{\oplus} \lambda T_{1}(\xi)+\mu T_{2}(\xi) d \nu(\xi), \lambda, \mu \in$ C, and $\mathcal{T}_{1} \mathcal{T}_{2}=\int^{\oplus} T_{1}(\xi) T_{2}(\xi) d \nu(\xi)$.

Proof. (a) Since the operator fields $\xi \mapsto T_{1}(\xi), \xi \rightarrow T_{2}(\xi)$ are decompositions of the operator $\mathcal{T}$ we have that a.e.

$$
T_{1}(\xi) x_{n}(\xi)=T_{2}(\xi) x_{n}(\xi) \text { for every } n \in \mathbf{N} \text {. }
$$

Since the operators $T_{1}(\xi), T_{2}(\xi)$ belong to $\mathcal{L}\left(\mathfrak{A}_{\xi}\right)$ a.e. we obtain from this that a.e.

$$
T_{1}(\xi) T x_{n}(\xi)=T_{2}(\xi) T x_{n}(\xi) \quad \text { for every } T \in \mathcal{L}\left(\mathfrak{A}_{\xi}\right)^{\prime}, n \in \mathbf{N} .
$$

Finally, by (1.5) this implies

$$
T_{1}(\xi)=T_{2}(\xi) \quad \text { a.e. }
$$

(b) The second assertion is an immediate consequence of the definition of natural decompositions.

1.4 TheOREM. For every $\mathcal{T} \in \mathcal{L}(\mathfrak{A})$ there exists a natural decomposition $\xi \mapsto T(\xi)$ of T such that

$$
\mathcal{T}^{*}=\int^{\oplus} T(\xi)^{*} d \nu(\xi) \text { and }\|T(\xi)\| \leq\|\mathcal{T}\| \text { a.e. }
$$

Proof. If we have $\mathcal{T}=\pi(\tilde{x})$ for some $x \in \Lambda$ then we obtain, for any $y \in \Lambda$,

$$
\pi(\tilde{x}) \tilde{y}=\tilde{x} \tilde{y}=\int^{\oplus} x(\xi) y(\xi) d \nu(\xi)=\int^{\oplus} \pi(x(\xi)) y(\xi) d \nu(\xi) .
$$

Since $\mathfrak{A}$ is dense in $\mathcal{H}$ the operator field $\xi \mapsto \pi(x(\xi))$ is a decomposition of $\pi(\tilde{x})$. By definition this decomposition is also a natural one and, by (1.3), $\|\pi(x(\xi))\| \leq\|\pi(\tilde{x})\|$ holds a.e. Clearly we also have $\pi(\tilde{x})^{*}=$ $\int^{\oplus} \pi(x(\xi))^{*} d \nu(\xi)$.

Now let $\mathcal{T} \in \mathcal{L}(\mathfrak{A})$ be given arbitrarily. By (1.5) the von Neumann algebra $\mathcal{L}(\mathfrak{A})$ is $\sigma$-finite. By Kaplansky's density theorem $\mathscr{T}$ belongs to the strong closure of the set $\{\delta \in \mathcal{L}(\mathfrak{U}) /\|\delta\| \leq\|\mathcal{T}\|\} \cap\{\pi(\tilde{x}) / x \in \Lambda\}$. Now it follows from the corollary in Dixmier [2], p. 31, that there exists a sequence $\left\{a_{n}\right\}_{n \in \mathbf{N}}$ in $\Lambda$ such that $\left\|\pi\left(\tilde{a}_{n}\right)\right\| \leq\|\mathcal{T}\|$ and $\left\{\pi\left(\tilde{a}_{n}\right)\right\}_{n \in \mathbf{N}}$ converges strongly to $\widetilde{\sigma}$. In particular

$$
\lim _{n \rightarrow \infty}\left\|\pi\left(\tilde{a}_{n}\right) \tilde{x}_{m}-\mathcal{T} \tilde{x}_{m}\right\|=0 \quad \text { for every } m \in \mathbf{N} .
$$


Let $\left\{y_{m}\right\}_{m \in \mathbf{N}}$ be a sequence in $\hat{\Lambda}$ such that

$$
\mathscr{T} \tilde{x}_{m}=\tilde{y}_{m}, \quad m \in \mathbf{N} .
$$

For every $m \in \mathbf{N}$ there exists a sequence $\left\{a_{n}^{(m)}\right\}_{n \in \mathbf{N}}$ in $\Lambda$ such that

$$
\begin{aligned}
& \left\{a_{n}^{(1)}\right\}_{n \in \mathbf{N}} \text { is a subsequence of }\left\{a_{n}\right\}_{n \in \mathbf{N}} ; \\
& \left\{a_{n}^{(m+1)}\right\}_{n \in \mathbf{N}} \text { is a subsequence of }\left\{a_{n}^{(m)}\right\}_{n \in \mathbf{N}}, \quad m \in \mathbf{N} ; \\
& \lim _{n \rightarrow \infty}\left\|\pi\left(a_{n}^{(m)}(\xi)\right) x_{m}(\xi)-y_{m}(\xi)\right\|=0 \quad \text { a.e., } \quad m \in \mathbf{N} .
\end{aligned}
$$

Hence there is a measurable subset $M \subset \Omega$ with $\nu(\Omega \backslash M)=0$ such that

$$
\lim _{n \rightarrow \infty}\left\|\pi\left(a_{n}^{(n)}(\xi)\right) x_{m}(\xi)-y_{m}(\xi)\right\|=0, \quad \xi \in M, m \in \mathbf{N} .
$$

From (1.3) we obtain

$$
\left\|\pi\left(a_{n}^{(n)}(\xi)\right)\right\| \leq\left\|\pi\left(a_{n}^{(n)}\right)\right\|, \quad \xi \in \Omega, n \in \mathbf{N} .
$$

Let $L$ be the linear subspace of $\prod_{\xi \in \Omega} \mathcal{F C}_{\xi}$ generated by all vector fields of the form $\xi \mapsto \pi^{\prime}\left(J_{\xi} x(\xi)\right) x_{m}(\xi)$, where $x \in \Lambda, m \in \mathbf{N}$. It follows from (1.4) that $L$ is a subspace of $\hat{\Lambda}$. By (1.5) the set $L_{\xi}=\{x(\xi) / / x \in L\}$ is a dense subspace of $\mathcal{H}_{\xi}$. From (1.6) we infer that for every $\xi \in M$ there is a linear operator $T_{0}(\xi)$ on $L_{\xi}$ such that

$$
\lim _{n \rightarrow \infty} \pi\left(a_{n}^{(n)}(\xi)\right) y=T_{0}(\xi) y, \quad y \in L_{\xi} .
$$

As $\left\|\pi\left(\tilde{a}_{n}^{(n)}\right)\right\| \leq\|\mathcal{T}\|$ holds for each $n \in \mathbf{N}$, we obtain from (1.7) that $T_{0}(\xi)$ is bounded and $\left\|T_{0}(\xi)\right\| \leq\|\mathscr{T}\|$. Since $L$ is dense in $\mathcal{H}_{\xi}$ there exists a (unique) bounded operator $T(\xi)$ on $\mathcal{H}_{\xi}$ which extends $T_{0}(\xi)$. Again we have $\|T(\xi)\| \leq\|\mathcal{T}\|$. Furthermore, the sequence $\left\{\pi\left(a_{n}^{(n)}(\xi)\right)\right\}_{n \in \mathbf{N}}$ converges strongly to $T(\xi)$. Hence $T(\xi)$ is contained in $\mathcal{L}\left(\mathfrak{A}_{\xi}\right)$. We set $T(\xi)=0$ for $\xi \in \Omega \backslash M$. By our construction of the operator field $\xi \mapsto T(\xi)$, for any $x \in L$ the vector field $\xi \mapsto T(\xi) x(\xi)$ is contained in $\hat{\Lambda}$. Moreover, from (1.4) and (1.6) we obtain that $\sigma \tilde{x}=\int^{\oplus} T(\xi) x(\xi) d \nu(\xi)$ holds for each $x \in L$. By (1.5) the set $\left\{\pi^{\prime}(J \tilde{x}) \tilde{x}_{m} / x \in \Lambda, m \in \mathbf{N}\right\}$ is total in $\mathcal{H}$. Therefore $\xi \mapsto T(\xi)$ is a (natural) decomposition of $\mathcal{T}$. As we have already shown, $\|T(\xi)\| \leq\|\widetilde{T}\|$ holds a.e.

If $\mathcal{T}$ is self-adjoint then it follows from Kaplansky's density theorem that the sequence $\left\{a_{n}\right\}_{n \in \mathbf{N}}$ above can be chosen so that $\pi\left(\tilde{a}_{n}\right)$ is self-adjoint for any $n \in \mathbf{N}$. Therefore, by our construction, $T(\xi)$ is the strong limit of a sequence of self-adjoint operators a.e. Hence $T(\xi)$ is self-adjoint a.e. From this we conclude that $\mathcal{T}=\int^{\oplus} T(\xi) d \nu(\xi)$ implies $\mathcal{T}^{*}=$ $\int^{\oplus} T(\xi)^{*} d \nu(\xi)$ for every $\mathcal{T} \in \mathcal{L}(\mathfrak{A})(\mathrm{cf} .1 .3(\mathrm{~b}))$. 
As a consequence of Theorem 1.4 and Proposition 1.3, one can show the following by using arguments similar to those used in Riedel [15], §2.

1.5 Proposition. Let $\mathcal{G}$ be a self-adjoint (not necessarily bounded) operator which is affiliated with $\mathcal{L}(\mathfrak{U})$. Then for any $\xi \in \Omega$ there is $a$ self-adjoint operator $G_{\xi}$ affiliated with $\mathfrak{L}\left(\mathfrak{A}_{\xi}\right)$ such that $f(\mathcal{G})=$ $\int^{\oplus} f\left(G_{\xi}\right) d \nu(\xi)$ holds for every bounded Borel measurable function on $\mathbf{R}$. The field $\xi \mapsto G_{\xi}$ is essentially unique. If $\mathcal{G}$ is regular or positive then $G_{\xi}$ is regular or positive a.e., respectively.

As another consequence of 1.4 we note the following:

1.6 Proposition. (a) If $\mathcal{L}(\mathfrak{U})$ is properly infinite then $\mathcal{L}\left(\mathfrak{U}_{\xi}\right)$ is properly infinite a.e. (cf. Dixmier [2], p. 206, Theorem 5).

(b) Suppose $\tau\left(\tau_{\xi}\right)$ is a trace on $\mathfrak{L}(\mathfrak{U})^{+}\left(\mathfrak{L}\left(\mathfrak{U}_{\xi}\right)^{+}, \xi \in \Omega\right)$ such that $\tau\left(\tau_{\xi}\right)$ is faithful (a.e.), and for any $\mathcal{T}=\int^{\oplus} T(\xi) d \nu(\xi) \in \mathcal{L}(\mathfrak{A})^{+}$, we have $\tau(\mathfrak{T})$ $=\int \tau_{\xi}(T(\xi)) d \nu(\xi)$. If $\mathfrak{L}(\mathfrak{U})$ is of type II then $\mathcal{L}\left(\mathfrak{U}_{\xi}\right)$ is of type II a.e. (cf. Riedel [13], Proposition 3.6).

2. Decomposition of the von Neumann algebras associated with KMS-states on $C^{*}$-algebras. Let $(\mathscr{Q}, \mathbf{R}, \boldsymbol{\sigma})$ be a $C^{*}$-dynamical system and assume the $C^{*}$-algebra $\mathscr{Q}$ contains a unit. Let us denote by $K(\mathbb{Q}, \sigma)$ the set of all KMS-states on $\mathbb{Q}$ (for the natural temperature $\beta=1$ ). The convex set $K(\mathbb{Q}, \sigma)$ is known to be a Choquet simplex with respect to the weak-* -topology (cf. Emch, Knops and Verboven [3], and Takesaki and Winnink [28]).

Let $\phi$ be an arbitrary element in $K(\mathbb{Q}, \sigma)$ and let $\left(\mathcal{H}_{\phi}, \pi_{\phi}\right)$ be the Gelfand-Naimark-Segal representation of $\mathscr{Q}$ associated with $\phi$. As $\mathbb{Q}$ has a unit there is a canonical cycle vector $e_{\phi}\left(\left\|e_{\phi}\right\|=1\right)$ such that

$$
\phi(T)=\left\langle\pi_{\phi}(T) e_{\phi}, e_{\phi}\right\rangle \text { for each } T \in \mathbb{Q} .
$$

From $\phi$ being a KMS-state it follows that $e_{\phi}$ is separating for $\pi_{\phi}(A)^{\prime \prime}$ (cf. Takesaki [25]). Moreover, the set $\mathfrak{U}_{\phi}=\left\{T e_{\phi} / T \in \pi_{\phi}(\mathscr{Q})^{\prime \prime}\right\}$ is an achieved left Hilbert algebra with $\mathcal{L}\left(\mathfrak{U}_{\phi}\right)=\pi_{\phi}(\mathscr{Q})^{\prime \prime}$. Let $\Delta_{\phi}$ be the modular operator associated with $\mathfrak{U}_{\phi}$. Then we have (cf. Takesaki [24], Theorem 13.2)

$$
\pi_{\phi}\left(\sigma_{t}(T)\right)=\Delta_{\phi}^{i t} \pi_{\phi}(T) \Delta_{\phi}^{-i t}, \quad T \in \mathbb{Q}, t \in \mathbf{R} .
$$

A state $\phi \in K(\mathbb{Q}, \phi)$ is an extreme point if and only if the representation $\pi_{\phi}$ is factorial, i.e. $\pi_{\phi}(\mathscr{Q})^{\prime \prime}$ is a factor. We shall denote the set of extreme points in $K(\mathbb{Q}, \sigma)$ by $\partial K(\mathbb{Q}, \sigma)$. 
Now we assume $K(\mathbb{Q}, \sigma)$ is non-empty and we fix an element $\psi \in$ $K(\Theta, \sigma)$. Let $\mu$ be the (unique) maximal measure (in the sense of Bishop and de Leeuw) with barycenter $\psi$ (cf. Alfsen [1]). Let $K_{\psi}(\mathbb{Q}, \sigma)$ be the set of all elements $\phi$ in $K(\mathbb{Q}, \sigma)$ such that the ideal $\left\{x \in \mathbb{Q} / \phi\left(x^{*} x\right)=0\right\}$ contains the kernel of the representation $\pi_{\psi} . K_{\psi}(\mathscr{Q}, \sigma)$ is a closed face of the Choquet simplex $K(\mathbb{Q}, \sigma)$. Hence $\mu / K_{\psi}(\mathbb{Q}, \sigma)$ is the maximal measure of $\psi$ on $K_{\psi}(Q, \sigma)$, and the set $\partial K_{\psi}(Q, \sigma)$ of all extreme points in $K_{\psi}(\Theta, \sigma)$ is equal to $K_{\psi}(\mathbb{Q}, \sigma) \cap \partial K(\mathbb{Q}, \sigma)$. In particular, $\mu$ is pseudo-concentrated on $\partial K_{\psi}(\mathbb{Q}, \sigma)$. We set $\Omega=\partial K_{\psi}(\mathbb{Q}, \sigma)$ and $\mathfrak{N}_{\phi}=\pi_{\phi}(\mathbb{Q})^{\prime \prime}$ for any $\phi \in K(\mathbb{Q}, \sigma)$.

Let $\Sigma_{0}$ be the $\sigma$-algebra of all subsets of $\Omega$ of the form $M \cap \Omega$, where $M$ is a Baire measurable subset of $K(\mathbb{Q}, \sigma)$. Since $\mu$ is pseudo-concentrated on $\Omega$ we can define a probability measure $\nu_{0}$ on $\Sigma_{0}$ by

$$
\nu_{0}(M \cap \Omega)=\mu(M),
$$

where $M$ is a Baire measurable subset of $K(\mathbb{Q}, \sigma)$. Let $(\Omega, \Sigma, \nu)$ be the completion of the measure space $\left(\Omega, \Sigma_{0}, \nu_{0}\right)$. As the measure $\mu$ coincides with the central measure of $\psi$ on $K(\mathbb{Q}, \sigma)$ (cf. Emch, Knops and Verboven [3]), we obtain from Sakai [17], 3.1.3, that there is a unique $*$-isomorphism $\hat{\Phi}$ from the center of $\Re_{\psi}$ onto $L^{\infty}(\Omega, \nu)$ such that

$$
\begin{aligned}
& \left(C \pi_{\psi}(T) e_{\psi}, e_{\psi}\right) \\
& \quad=\int \hat{\Phi}(C)(\phi) \phi(T) d \nu(\phi), \quad C \in \mathfrak{M}_{\psi} \cap \mathfrak{K}_{\psi}^{\prime}, T \in \mathbb{Q} .
\end{aligned}
$$

Let $\Lambda_{0}$ be the set of all vector fields on $K(\mathbb{Q}, \sigma)$ of the form $\phi \rightarrow \pi_{\phi}(T) e_{\phi}$, $T \in \mathbb{Q}$, and let $\Lambda=\left\{x_{/ \Omega} / x \in \Lambda_{0}\right\}$. For every $T \in \mathbb{Q}$ the function $\phi \rightarrow$ $\left\|\pi_{\phi}(T) e_{\phi}\right\|\left(=\phi\left(T^{*} T\right)^{1 / 2}\right)$ is bounded and measurable with respect to the measurable space $(\Omega, \Sigma)$. Hence, as in $\S 1$ we can associate with $\Lambda$ an integrable field of Hilbert spaces $\left(\left\{\mathcal{H}_{\phi}\right\}_{\phi \in \Omega}, \hat{\Lambda}\right)$. From (2.2) we infer that there exists a unique isomorphism from $\mathcal{H}_{\psi}$ onto $\int^{\oplus \mathcal{K}_{\phi}} d \nu(\phi)$ which maps $\pi_{\psi}(T) e_{\psi}$ onto $\int^{\oplus} \pi_{\phi}(T) e_{\phi} d \nu(\phi)$ for any $T \in \mathbb{Q}$. Thus we may write

$$
\mathcal{H}_{\psi}=\int^{\oplus} \mathcal{H}_{\phi} d \nu(\phi)
$$

Let $\mathfrak{A}=\{\tilde{x} / x \in \Lambda\}$. The following proposition shows that the theory of $\S 1$ is applicable to our present situation.

2.1 Proposition. $\left(\left\{\mathfrak{U}_{\phi}\right\}_{\phi \in \Omega}, \Lambda\right)$ is an integrable field of left Hilbert algebras. $\mathfrak{U}$ is a left Hilbert subalgebra of $\mathfrak{U}_{\psi}$ satisfying $\mathfrak{U}^{\prime \prime}=\mathfrak{U}_{\psi}$. 
Proof. We have to show that the set $\Lambda$ satisfies conditions (1.1)-(1.5). Clearly, $\Lambda$ satisfies (1.1) and (1.2). Theorem 3.1 in Riedel [15] states that the theory of topological direct integrals of left Hilbert algebras can be applied to the present situation. If $\phi \mapsto x(\phi)$ is a continuous vector field on $K(\Theta, \sigma)$, then it follows from Riedel [12], 1.1(3), that $x$ can be approximated uniformly on $K(\mathbb{Q}, \sigma)$ by linear combinations of vector fields of the form $\phi \rightarrow f(\phi) \pi_{\phi}(a) e_{\phi}$, where $a \in \mathbb{Q}$ and $f$ is an arbitrary continuous function on $K(\mathbb{Q}, \sigma)$. Hence the vector field $\Omega \ni \phi \mapsto x(\phi)$ can be approximated uniformly by elements of $\hat{\Lambda}$. Therefore $x_{/ \Omega}$ belongs to $\hat{\Lambda}$ (cf. Wils [32], 2.3). It follows that $\phi \rightarrow J_{\phi} x(\phi)$ belongs to $\hat{\Lambda}$ for each $x \in \hat{\Lambda}$. Moreover, by Riedel [15], 1.7,

$$
\begin{aligned}
\left(J_{\psi} \pi_{\psi}(a) e_{\psi}, \pi_{\psi}(b) e_{\psi}\right) & =\int\left(J_{\phi} \pi_{\phi}(a) e_{\phi}, \pi_{\phi}(b) e_{\phi}\right) d \mu(\phi) \\
& =\int_{\Omega}\left(J_{\phi} \pi_{\phi}(a) e_{\phi}, \pi_{\phi}(b) e_{\phi}\right) d \nu(\phi)
\end{aligned}
$$

holds for each $a, b \in \mathbb{Q}$. This shows that $\phi \rightarrow J_{\phi}$ is a decomposition of $J_{\psi}$, i.e. (1.4) is satisfied.

Since $e_{\phi}$ is a unit in $\mathfrak{A}_{\phi}$ for every $\phi \in K(\mathbb{Q}, \sigma)$ and $e_{\psi}=\int^{\oplus} e_{\phi} d \nu(\phi)$ holds, condition (1.5) is satisfied.

By Strătilă and Zsidó [20], 10.5, $\mathfrak{A}$ is a left Hilbert subalgebra of $\mathfrak{A}_{\psi}$ with $\mathfrak{U}^{\prime \prime}=\mathfrak{A}_{\psi}$. In particular, $\mathfrak{A}$ is dense in $\mathcal{H}_{\psi}$. Therefore it remains to show that $\|\pi(x(\phi))\| \leq\|\pi(\tilde{x})\|$ holds for every $x \in \Lambda, \phi \in \Omega$. Let $T \in \mathbb{Q}$. Then we have $\pi\left(\pi_{\phi}(T) e_{\phi}\right)=\pi_{\phi}(T)$ for every $\phi \in K(\mathbb{Q}, \sigma)$. By the definition of $\Omega$, for each $\phi \in \Omega$ the operator $\pi_{\phi}(T)$ is the image of the operator $\pi_{\psi}(T)$ with respect to some $*$-homomorphism from $\pi_{\psi}(\mathbb{Q})$ onto $\pi_{\phi}(Q)$. Since every $*$-homomorphism of $C^{*}$-algebras is norm decreasing we obtain

$$
\begin{aligned}
\left\|\pi\left(\pi_{\phi}(T) e_{\phi}\right)\right\| & =\left\|\pi_{\phi}(T)\right\| \leq\left\|\pi_{\psi}(T)\right\|=\left\|\pi\left(\pi_{\psi}(T)\right) e_{\psi}\right\| \\
& =\left\|\pi\left(\int^{\oplus} \pi_{\phi}(T) e_{\phi}\right)\right\| .
\end{aligned}
$$

This shows that condition (1.3) is also satisfied.

Next we want to show that there is also a disintegration of the crossed product of the von Neumann algebra $\mathscr{L}(\mathfrak{U})=\mathfrak{N}_{\psi}$ by the corresponding modular action, and this disintegration is closely related to the disintegration of $\mathscr{L}(\mathfrak{U})$. For any $\phi \in K(\mathbb{Q}, \sigma)$ and $t \in \mathbf{R}$ we define an automorphism $\sigma_{t}^{\phi}$ of the $C^{*}$-algebra $\mathbb{Q}_{\phi}=\pi_{\phi}(\mathscr{Q})$ as follows:

$$
\sigma_{t}^{\phi}\left(\pi_{\phi}(T)\right)=\pi_{\phi}\left(\sigma_{t}(T)\right), \quad T \in \mathbb{Q} .
$$


$\left(\mathscr{Q}_{\phi}, \mathbf{R}, \boldsymbol{\sigma}^{\phi}\right)$ is a $C^{*}$-dynamical system. For any $\phi \in K(\mathbb{Q}, \boldsymbol{\sigma})$ let $\left\{\overline{\boldsymbol{\sigma}}_{t}^{\phi}\right\}_{t \in \mathbf{R}}$ be the modular automorphism group associated with $\mathfrak{A}_{\phi}$. By (2.1) we have $\bar{\sigma}_{t}^{\phi}(T)=\sigma_{t}^{\phi}(T)$ for $T \in \mathbb{Q}_{\phi}, \phi \in K(\mathbb{Q}, \sigma), t \in \mathbf{R}$. For any $\phi \in K(\mathbb{Q}, \sigma)$ we associate to the crossed product $\mathfrak{K}_{\phi} \otimes_{\bar{\sigma}^{\phi}} \mathbf{R}$ a left Hilbert algebra $\mathfrak{B}_{\phi}$ as in Riedel [16], §2, so $\mathcal{L}\left(\mathfrak{B}_{\phi}\right)=\mathfrak{N}_{\phi} \otimes_{\bar{\sigma}^{\phi}} \mathbf{R}$ holds. We set $\mathcal{L}_{\phi}=L^{2}\left(\mathbf{R}, \mathcal{H}_{\phi}\right)$. For any $\phi \in K(\mathbb{Q}, \boldsymbol{\sigma}), t \in \mathbf{R}$, we set $\mathfrak{A}_{(\phi, t)}=\mathfrak{U}_{\phi}$. Let $\mathfrak{R}$ be the smallest linear subspace of $\prod_{(\phi, t) \in K(\mathbb{Q}, \sigma) \otimes \mathbf{R}} \mathcal{L}\left(\mathfrak{A}_{(\phi, t)}\right)$ which satisfies the following two conditions:

(2.4) For any $T \in \mathbb{Q}$ and $f \in C_{c}(\mathbf{R})$ the operator field $(\phi, t) \mapsto$ $f(t) \pi_{\phi}(T)$ belongs to $\Re$.

(2.5) If the operator fields $(\phi, t) \mapsto T_{1}(\phi, t)$ and $(\phi, t) \mapsto T_{2}(\phi, t)$ belong to $\Re$ then the same is true for the operator fields $(\phi, t) \mapsto$ $\int \sigma_{s}^{\phi}\left(T_{1}(\phi, s+t)\right) T_{2}(\phi,-s) d s$ and $(\phi, t) \mapsto \sigma_{-t}^{\phi}\left(T_{1}(\phi,-t)\right)^{*}$

It follows from the proof of Riedel [16], 3.5(a), that for any operator field $(\phi, t) \rightarrow T(\phi, t)$ in $\Re$ and $\phi \in \Omega$ the mapping $t \mapsto T(\phi, t)$ is a continuous function with compact support from $\mathbf{R}$ into $\mathcal{Q}_{\phi}$. In particular, the integrals which occur in (2.5) are well defined. Let $\Gamma_{0}$ be the set of all vector fields $\phi \rightarrow x(\phi)$ in $\Pi_{\phi \in K(\mathbb{Q}, \sigma)} \mathcal{L}_{\phi}$ such that $x(\phi)(t)=T(\phi, t) e_{\phi}$, $t \in \mathbf{R}$, for some $T \in \mathscr{R}$, and let $\Gamma=\left\{x_{/ \Omega} / x \in \Gamma_{0}\right\}$. It follows from Riedel [16], 3.5(a), that for every vector field $x$ in $\Gamma$ the function $\phi \mapsto\|x(\phi)\|$ is bounded and measurable with respect to the measurable space $(\Omega, \Sigma)$. Let $\left(\left\{\mathcal{L}_{\phi}\right\}_{\phi \in \Omega}, \hat{\Gamma}\right)$ be the integrable field of Hilbert spaces associated with $\Gamma$ as in $\S 1$. From (2.3), as well as from Riedel [16], 3.3., we infer that there is a unique isomorphism from $\varrho_{\psi}$ onto $\int^{\oplus} \mathcal{L}_{\phi} d \nu(\phi)$ which maps $x(\psi)$ onto $\int^{\oplus} x_{/ \Omega}(\phi) d \nu(\phi)$ for every $x \in \Gamma_{0}$. Therefore we may write

$$
\varrho_{\psi}=\int^{\oplus} \mathcal{L}_{\phi} d \nu(\phi) \text {. }
$$

Let $\mathfrak{B}=\{x / x \in \Gamma\}$. We can now prove an analogue of 2.1 for crossed products.

2.2 Proposition. $\left(\left\{\mathfrak{B}_{\phi}\right\}_{\phi \in \Omega}, \Gamma\right)$ is an integrable field of left Hilbert algebras. $\mathfrak{B}$ is a left Hilbert subalgebra of $\mathfrak{B}_{\psi}$ with $\mathfrak{B}^{\prime \prime}=\mathfrak{B}_{\psi}^{\prime \prime}$.

Proof. By the construction of $\Re$ and $\Gamma$, conditions (1.1) and (1.2) are satisfied. It follows from Riedel [16], 3.5(a), that (1.4) is valid (see also the proof of 2.1). From Riedel [16], 3.5(b), we obtain that (1.5) is satisfied.

Since $Q_{\psi}$ is dense in $\pi_{\psi}(\mathscr{Q})^{\prime \prime}$ with respect to the $s^{*}$-topology, the set $\left\{\pi_{\psi}(T) e_{\psi} / T \in \mathbb{Q}\right\}$ is a left Hilbert subalgebra equivalent to $\mathfrak{A}_{\psi}$. Since $\mathfrak{B}$ contains the set $\left\{t \rightarrow f(t) \pi_{\psi}(T) e_{\psi} / f \in C_{c}(\mathbf{R}), T \in \mathbb{Q}\right\}$, it follows that $\mathfrak{B}$ is dense in $\mathfrak{B}_{\psi}$ with respect to the \#-norm (cf. Riedel [16], 2.1). As $\mathfrak{B}$ is an 
involutive subalgebra of $\mathfrak{B}_{\psi}$, it follows that $\mathfrak{B}$ is a left Hilbert algebra with $\mathfrak{B}^{\prime \prime}=\mathfrak{B}_{\psi}^{\prime \prime}$ (cf. Strătilă and Szidó [20], 10.5). In particular, $\mathfrak{B}$ is dense in $\mathfrak{B}_{\psi}$. Hence it remains to prove that $\|\pi(x(\phi))\| \leq\|\pi(\tilde{x})\|$ holds for $x \in \Gamma$, $\phi \in \Omega$. For any $\phi \in K(\mathbb{Q}, \sigma)$ let $\delta_{\phi}$ be the regular representation of the crossed product $Q_{\phi} \otimes_{\phi} \mathbf{R}$ which is induced by $\left(1_{\mathbb{Q}_{\phi}}, \mathcal{H}_{\phi}\right)$ (cf. Pedersen 10]). Then $\delta_{\phi}$ is faithful (cf. Pedersen [10], 7.7.5, 7.7.7). Moreover, we have $T(\phi, t) \in \mathbb{Q}_{\phi}$ for $(\phi, t) \in K(\mathbb{Q}, \sigma) \times \mathbf{R}$, and $T(\phi, \cdot) \in \mathbb{Q}_{\phi} \otimes_{\phi} \mathbf{R}$ for $T \in$ $\Re$. We shall need the following identity (cf. Riedel [16], §2):

$$
\delta_{\phi}(T(\phi, \cdot))=\pi\left(T(\phi, \cdot) e_{\phi}\right) \quad \text { for } T \in \Re, \phi \in K(\Theta, \sigma) .
$$

For every $\phi \in \Omega$ there is a unique homomorphism $\rho_{\phi}$ from $\mathbb{Q}_{\psi}$ onto $\mathbb{Q}_{\phi}$ such that $\rho_{\phi} \circ \pi_{\psi}=\pi_{\phi}$. Pedersen [10], 7.6.4, states that for each $\phi \in \Omega$ there is a (non-degenerated) representation, say $L_{0}^{(\phi)}$, of $L^{1}\left(\mathbf{R}, \mathcal{H}_{\psi}\right)$ on $L^{2}\left(\mathbf{R}, \mathcal{H}_{\phi}\right)$ such that

$$
L_{0}^{(\phi)}(T)=\int \lambda^{(\phi)}(t) \pi_{\sigma^{\phi}} \circ \rho_{\phi}(T(t)) d t \quad \text { for } T \in L^{1}\left(\mathbf{R}, \mathbb{Q}_{\psi}\right)
$$

(where $\lambda^{(\phi)}(t) x(s)=x(s-t)$ for $\left.x \in C_{c}\left(\mathbf{R}, \mathcal{H}_{\phi}\right)\right), \quad\left(\pi_{\sigma^{\phi}}(T) x\right)(t)=$ $\sigma_{-t}^{\phi}(T) x(t)$ for $\left.T \in \mathbb{Q}_{\phi}, x \in C_{c}\left(\mathbf{R}, \mathcal{H}_{\phi}\right)\right)$. By the definition of the regular representation, the algebra $L_{0}^{(\phi)}\left(L^{1}\left(\mathbf{R}, \mathbb{Q}_{\psi}\right)\right)$ is contained in $\delta_{\phi}\left(Q_{\phi} \otimes_{\sigma^{\phi}} \mathbf{R}\right)$. Since $\rho_{\phi}$ is onto, $L_{0}^{(\phi)}\left(L^{1}\left(\mathbf{R}, Q_{\psi}\right)\right)$ is dense in $\delta_{\phi}\left(Q_{\phi} \otimes_{\sigma^{\phi}} \mathbf{R}\right)$. By Pedersen [10], 7.6.6, the mapping $L_{0}^{(\psi)}$ can be (uniquely) extended to a $*$-homomorphism of $\mathbb{Q}_{\psi} \otimes_{\sigma^{\psi}} \mathbf{R}$ onto $\delta_{\phi}\left(\mathbb{Q}_{\phi} \otimes_{\sigma^{\phi}} \mathbf{R}\right)$. Since $\delta_{\psi}$ is faithful we can define a * -homomorphism $L$ from $\delta_{\psi}\left(\mathbb{Q}_{\psi} \otimes_{\sigma^{\psi}} \mathbf{R}\right)$ onto $\delta_{\phi}\left(\mathbb{Q}_{\phi} \otimes_{\sigma^{\phi}} \mathbf{R}\right)$ such that

$$
L_{\phi}\left(\delta_{\psi}(T(\psi, \cdot))\right)=L_{0}^{(\phi)}(T(\psi, \cdot))=\delta_{\phi}\left(\rho_{\phi}(T(\psi, \cdot))\right)
$$

for each $T \in \mathcal{R}$. However, by the definition of $\rho_{\phi}$, and since $\rho_{\phi} \circ \sigma_{t}^{\psi}=$ $\sigma_{t}^{\phi} \circ \rho_{\phi}$ holds, we must have

$$
\rho_{\phi}(T(\psi, t))=T(\phi, t), \quad t \in \mathbf{R},
$$

for each $T \in \mathcal{R}$ and $\phi \in \Omega$. From this, as well as from (2.7), we conclude that

$$
\|\pi(x(\phi))\| \leq\|\pi(x(\psi))\| \quad \text { for } x \in \Gamma_{0}, \phi \in \Omega .
$$

By (2.6) we have $\pi(x(\psi))=\pi\left(\widetilde{x_{/ \Omega}}\right)$ for every $x \in \Gamma_{0}$. Thus we have shown that condition (1.3) is also satisfied.

We note the following result on the disintegration of relatively invariant traces (cf. Takesaki [26]). Its proof runs parallel with the proof of Riedel [16], Proposition 3.9. 
2.3 Proposition. For every $\phi \in K(\mathbb{Q}, \sigma)$ there exists a faithful relatively invariant trace $\tau_{\phi}$ on $\mathcal{L}\left(\mathfrak{B}_{\phi}\right)^{+}$such that for any $\mathcal{T}=\int^{\oplus} T(\phi) d \nu(\phi) \in$ $\mathcal{L}\left(\mathfrak{B}_{\psi}\right)^{+}$, the function $\phi \mapsto \tau_{\phi}(T(\phi))$ is measurable on $\Omega$ and $\tau_{\psi}(\mathcal{T})=$ $\int \tau_{\phi}(T(\phi)) d \nu(\phi)$ holds.

As a first application of our theory we prove the following:

2.4 Corollary. If $\Re_{\psi}$ is a continuous von Neumann algebra then $\mathfrak{K}_{\phi}$ is continuous a.e.

Proof. From van Daele [31], Part II, 4.1 and 4.2, we obtain that if $\mathscr{Z}$ is a semifinite central projection in $\mathfrak{R}_{\psi}$ then

$$
\mathcal{L}\left(\mathfrak{B}_{\psi}\right)_{\mathscr{Q} \otimes 1} \cong\left(\Re_{\psi}\right)_{\mathscr{Q}} \otimes L^{\infty}(\mathbf{R}) \text {. }
$$

Similarly, if $\mathfrak{R}_{\phi}$ is semifinite for some $\phi \in \Omega$ then

$$
\mathcal{L}\left(\mathfrak{B}_{\phi}\right) \cong \mathfrak{K}_{\phi} \otimes L^{\infty}(\mathbf{R}) \text {. }
$$

As $\mathcal{L}\left(\mathfrak{B}_{\phi}\right)$ is semifinite for every $\phi \in K(\mathscr{Q}, \sigma)$, we obtain from this, as well as from van Daele [31], Part II. 4.7, that $\Re_{\psi}\left(\Re_{\phi}\right)$ is continuous if and only if $\mathscr{L}\left(\mathfrak{B}_{\psi}\right)\left(\mathfrak{L}\left(\mathfrak{B}_{\phi}\right), \phi \in \Omega\right)$ is of type II. Therefore our assertion follows immediately from $1.6(\mathrm{~b})$ and 2.3 .

3. Reduction of von Neumann algebras with cyclic and separating vectors. Let $\Re$ be a von Neumann algebra which acts on the Hilbert space $\mathcal{H}$ with a cyclic and separating vector $e$. We shall associate with $\Re$ a $C^{*}$-dynamical system in such a manner that the theory of $\S 2$ yields a decomposition of $\mathfrak{T}$ into factors. Let $\bar{\psi}$ be the vector state on $\Re$ associated with $e$ and let $\left\{\overline{\boldsymbol{\sigma}}_{t}\right\}_{t \in \mathbf{R}}$ be the corresponding modular automorphism group. Let $\mathbb{Q}$ be the set of all elements $T$ in $\Re$ such that the function $t \mapsto \bar{\sigma}_{t}(T)$ from $\mathbf{R}$ into $\Re$ is continuous, where $\Re$ is equipped with the norm topology. $\mathbb{Q}$ is a $C^{*}$-algebra which is invariant under the modular automorphism $\bar{\sigma}_{t}, t \in \mathbf{R}$. Let $\psi$ be the vector state on $\mathscr{Q}$ associated with $e$, and for any $t \in \mathbf{R}$ let $\sigma_{t}$ be the restriction of the automorphism $\bar{\sigma}_{t}$ to $Q$. Then we have the following.

(3.1) $Q$ is weakly dense in $\mathscr{N}$ and $\mathscr{Q}$ contains the fixed-point algebra $\Re^{\bar{\sigma}}$ of $\Re$ with respect to the automorphisms $\overline{\boldsymbol{\sigma}}_{t}, t \in \mathbf{R}$ (cf. Pedersen [1], 7.5.1). Moreover, $(\mathbb{Q}, \mathbf{R}, \sigma)$ is a $C^{*}$-dynamical system and $\psi$ is a KMS-state on $\mathbb{Q}$.

This shows us that the theory of $\S 2$ can be applied to our present situation. Henceforth we shall retain the notation we have introduced in $\S 2$. So we may identify $\Re$ and $\Re_{\psi}$. 
3.1 TheOREM. Suppose $\tau$ is a faithful trace on $\mathfrak{T}^{+}$. Then for every $\phi \in \Omega$ there exists a trace $\tau_{\phi}$ on $\mathfrak{M}_{\phi}^{+}$which is faithful a.e. such that for any

$$
\mathcal{T}=\int^{\oplus} T(\phi) d \nu(\phi) \in \mathfrak{T}^{+} \text {we have } \tau(\mathcal{T})=\int \tau_{\phi}(T(\phi)) d \nu(\phi) .
$$

Proof. By Pedersen and Takesaki [11], 51.2, there exists a regular positive self-adjoint operator $\mathcal{G}$ affiliated with $\mathfrak{\Re}^{\bar{\sigma}}$ such that $\mathcal{G}^{-1}$ is the Radon-Nikodym derivate of $\tau$ with respect to $\bar{\psi}$, i.e. $\tau=\bar{\psi}\left(\mathcal{G}^{-1}\right)$ (cf. Pedersen and Takesaki [11], p. 62). More precisely, the following is true. For any $n \in \mathbf{N}$ we define a function $f_{n}$ on $\mathbf{R}$ by

$$
f_{n}(t)= \begin{cases}t(1+t / n)^{-1} & \text { if } t \in \mathbf{R}^{+}, \\ 0 & \text { elsewhere. }\end{cases}
$$

For every $n \in \mathbf{N}$ the operator $f_{n}\left(\mathcal{G}^{-1}\right)$ is bounded. We define a positive functional $\tau^{(n)}$ on $\Re$ by

$$
\tau^{(n)}(\mathcal{T})=\bar{\psi}\left(f_{n}\left(\mathcal{G}^{-1}\right) \mathcal{T}\right), \quad \mathcal{T} \in \Re .
$$

For any $\mathcal{T} \in \mathfrak{T}^{+}$the sequence $\left\{\boldsymbol{\tau}^{(n)}(\mathcal{T})\right\}_{n \in \mathbf{N}}$ is monotonely increasing and we have

$$
\lim _{n \rightarrow \infty} \tau^{(n)}(\mathcal{T})=\tau(\mathcal{T}) .
$$

For every $t \in \mathbf{R}$ we set $\mathscr{Q}_{t}=\mathcal{G}^{t t}$. Since $\tau$ is a trace we infer from Pedersen and Takesaki [11], 4.6, that the one parameter group $\left\{\mathscr{U}_{t}\right\}_{t \in \mathbf{R}}$ implements the modular automorphism group $\left\{\sigma_{t}\right\}_{t \in \mathbf{R}}$, i.e.

$$
\mathscr{U}_{t} \sigma \mathcal{U}_{t}^{*}=\bar{\sigma}_{t}(\mathcal{T}) \text { for } \mathcal{T} \in \mathfrak{T}, t \in \mathbf{R} .
$$

In particular, $\mathscr{Q}_{t}$ belongs to the fixed-point algebra $\Re^{\bar{\sigma}}$ for every $t \in \mathbf{R}$. By (3.1) $\mathcal{U}_{t}$ belongs to $\mathcal{Q}$. Therefore we obtain

$$
\begin{gathered}
\mathscr{U}_{t}=\int^{\oplus} \pi_{\phi}\left(\mathscr{U}_{t}\right) d \nu(\phi), \quad t \in \mathbf{R} ; \\
\bar{\sigma}_{t}^{\phi}(T)=\pi_{\phi}\left(\mathscr{U}_{t}\right) T \pi_{\phi}\left(\mathscr{U}_{t}\right)^{*}, \quad T \in \Re_{\phi}, t \in \mathbf{R} .
\end{gathered}
$$

It follows from 1.5 that for every $\phi \in \Omega$ there exists a regular positive self-adjoint operator $G_{\phi}$ affiliated with $\Re_{\phi}$ such that

$$
\mathcal{Q}_{t}=\int^{\oplus} G_{\phi}^{t t} d \nu(\phi), \quad t \in \mathbf{R} .
$$

By (3.2) we obtain from this that for each $t \in \mathbf{R}$,

$$
G_{\phi}^{i t}=\pi_{\phi}\left(\mathscr{Q}_{t}\right) \text { a.e. }
$$


Hence by (3.3) there exists a measurable subset $M \subset \Omega$ with $\nu(\Omega \backslash M)=0$ such that

$$
\bar{\sigma}_{t}^{\phi}(T)=G_{\phi}^{i t} T G_{\phi}^{-i t}, \quad G \in \Re_{\phi}, \phi \in M, t \in \mathbf{Q},
$$

Q being the set of rational numbers. Since the functions $t \mapsto \bar{\sigma}_{t}^{\phi}(T)$ and $t \mapsto G_{\phi}^{i t} T G_{\phi}^{-i t}$ are strongly continuous for every $T \in \mathfrak{N}_{\phi}$, we conclude from this that

$$
\overline{\boldsymbol{\sigma}}_{t}^{\phi}(T)=G_{\phi}^{i t} T G_{\phi}^{-i t}, \quad T \in \mathfrak{N}_{\phi}, \phi \in M, t \in \mathbf{R} .
$$

This means that the one parameter group $\left\{G_{\phi}^{t t}\right\}_{t \in \mathbf{R}}$ implements the modular automorphism group $\left\{\bar{\sigma}_{t}^{\phi}\right\}_{t \in \mathbf{R}}$. Next, for any $\phi \in M, n \in \mathbf{N}$, we define a positive functional $\tau_{\phi}^{(n)}$ on $\Re_{\phi}$ by

$$
\tau_{\phi}^{(n)}(T)=\phi\left(f_{n}\left(G_{\phi}^{-1}\right) T\right), \quad T \in \Re_{\phi} .
$$

For any $T \in \mathscr{T}_{\phi}$ the sequence $\left\{\tau_{\phi}^{(n)}(T)\right\}_{n \in \mathbf{N}}$ is monotone increasing and by the proof of Pedersen and Takesaki [11], 7.4, there exists a faithful trace $\tau_{\phi}$ on $\Re_{\phi}^{+}$such that

$$
\lim _{n \rightarrow \infty} \tau_{\phi}^{(n)}(T)=\tau_{\phi}(T), \quad T \in \mathfrak{M}_{\phi}^{+} .
$$

For convenience we set $\tau_{\phi}=0$ if $\phi \in \Omega \backslash M$. By 1.5 we have

$$
f_{n}\left(\mathcal{G}^{-1}\right)=\int^{\oplus} f_{n}\left(G_{\phi}^{-1}\right) d \nu(\phi) .
$$

Therefore we obtain for every $\mathcal{T}=\int^{\oplus} T(\phi) d \nu(\phi)$,

$$
\tau^{(n)}(\mathcal{T})=\int \tau_{\phi}^{(n)}(T(\phi)) d \nu(\phi), \quad n \in \mathbf{N}
$$

Moreover, the function $\phi \rightarrow \tau_{\phi}(T(\phi))$ is measurable, and from Lebesgue's monotone convergence theorem we conclude

$$
\begin{aligned}
\tau(\mathcal{T}) & =\lim _{n \rightarrow \infty} \tau^{(n)}(\mathcal{T})=\lim _{n \rightarrow \infty} \int \tau^{(n)}(T(\phi)) d \nu(\phi) \\
& =\int \lim _{n \rightarrow \infty} \tau_{\phi}^{(n)}(T(\phi)) d \nu(\phi)=\int \tau_{\phi}(T(\phi)) d \nu(\phi) .
\end{aligned}
$$

3.2 CoROllaRy. If $\mathfrak{T}$ is of type $\mathrm{II}_{\infty}$ or type $\mathrm{II}_{1}$ then $\mathfrak{T l}_{\phi}$ is of type $\mathrm{II}_{\infty}$ or type $\mathrm{II}_{1}$, respectively, a.e.

Proof. If $\mathscr{N}$ is of type $\mathrm{II}_{\infty}$ then it follows from 1.6 and 3.1 that $\mathscr{T}_{\phi}$ is of type $\mathrm{II}_{\infty}$ a.e. 
If $\Re$ is of type $\mathrm{II}_{1}$ then the trace $\tau$ in 3.1 can be chosen to be finite. As $\tau\left(1_{\mathcal{H}}\right)=\int \tau_{\phi}\left(1_{\mathcal{F}_{\phi}}\right) d \nu(\phi)<\infty$ holds, $\tau_{\phi}$ is a finite trace a.e. Since $\tau_{\phi}$ is faithful a.e. $\mathfrak{N}_{\phi}$ must be finite a.e. Therefore we obtain from 1.6 that $\mathfrak{N}_{\phi}$ is of type $\mathrm{II}_{1}$ a.e.

If $\Re$ is a finite von Neumann algebra then it is more convenient to choose a Hilbert space $\mathcal{H}$ and the cyclic and separating vector $e$ in such a manner that $\bar{\psi}$ is a trace. In this case all left Hilbert algebras which occur are in fact Hilbert algebras and we can apply the theory in Riedel [13] or [14].

If $\Re$ is of type $I_{\infty}$ then we do not know whether the factors $\Re_{\phi}$ are of type $I_{\infty}$ or not in general. However, if we carry out some slight modifications then this case can also be settled. For convenience we only consider the case that $\Re$ is homogeneous of type $I_{n}$ for $n \in \mathbf{N} \cup\{\infty\}$. By Sakai [17], 2.3.3, we may assume $\mathscr{N}$ is the tensor product of an abelian von Neumann algebra $\mathcal{C}$ and a type $I_{n}$ factor $\Re$, i.e. $\Re=\bigodot \otimes \Re$. We may assume the state $\bar{\psi}$ is a tensor product of a faithful normal state $\bar{\psi}_{1}$ on $\bigodot$ and a faithful normal state $\bar{\psi}_{2}$ on $\mathscr{B}$. Let $\mathscr{K}$ denote the $C^{*}$-subalgebra of $\mathscr{B}$ generated by the compact operators and by the unit of $\mathscr{B}$ (so that $\mathscr{K}=\mathscr{B}$ if $n<\infty)$, and let $A_{0}$ be the $C^{*}$-tensor product of $\mathcal{C}$ and $\mathcal{K}$.

3.3 Proposition. $\mathbb{Q}_{0}$ is a type I $C^{*}$-subalgebra of $\mathbb{Q} . \mathbb{Q}_{0}$ is $\sigma$-weakly dense in $\Re$ and invariant under the automorphisms $\overline{\boldsymbol{\sigma}}_{t}, t \in \mathbf{R}$.

Proof. Since $\mathcal{Q}_{0}$ is the $C^{*}$-tensor product of a type I $C^{*}$-algebra and an abelian $C^{*}$-algebra, it is also of type $\mathrm{I}$. As $\Re$ is semifinite there exists a strongly continuous one parameter group $\left\{\mathcal{Q}_{t}\right\}_{t \in \mathbf{R}}$ of unitaries in $\mathscr{B}$ which implements the modular automorphism group associated with $\bar{\psi}_{2}$. Then the modular automorphism group $\left\{\sigma_{t}\right\}_{t \in \mathbf{R}}$ associated with $\bar{\psi}$ is implemented by $\left\{1 \otimes \mathscr{Q}_{t}\right\}_{t \in \mathbf{R}}$. Since $\mathcal{K}$ is invariant under the inner automorphisms induced by $\mathscr{Q}_{t}$ we obtain that $\mathbb{Q}_{0}$ is invariant under the automorphisms $\bar{\sigma}_{t}, t \in \mathbf{R}$. It is clear that $\mathbb{Q}_{0}$ is $\sigma$-weakly dense in $\mathfrak{T}$. In order to verify that $\mathbb{Q}_{0}$ is contained in $\mathbb{Q}$ we have to show that the function $t \mapsto \bar{\sigma}_{t}(\mathcal{T})$ is norm continuous for every $\mathcal{T} \in \mathbb{Q}_{0}$. Since the function $t \mapsto 1 \otimes \mathcal{Q}_{t}$ is strongly continuous, the function $t \mapsto\left(1 \otimes \mathcal{Q}_{t}\right) \mathscr{T}\left(1 \otimes \mathcal{U}_{t}\right)^{*}$ must be norm continuous for every operator $\mathcal{T}=1 \otimes \mathscr{P}$, where $\mathcal{P}$ is a one-dimensional projection in $\mathcal{K}$. Hence this function is also continuous for every operator $\mathcal{T}=1 \otimes 2$, where $\mathcal{Q}$ is an operator in $\mathcal{K}$ of finite rank. As the set of operators of finite rank is uniformly dense in $\mathscr{K}$, we obtain from this that $t \rightarrow\left(1 \otimes \mathcal{Q}_{t}\right) \mathscr{T}\left(1 \otimes \mathcal{Q}_{t}\right)^{*}$ is norm continuous for every 
$\mathcal{T} \in 1 \otimes \mathcal{K}$. Hence $t \rightarrow\left(1 \otimes \mathcal{U}_{t}\right) \mathcal{T}\left(1 \otimes \mathcal{U}_{t}\right) *$ is norm continuous for every $\tilde{T} \in \mathbb{Q}_{0}$.

3.4 COROLLARY. If we replace the $C^{*}$-algebra $Q$ by $\mathbb{Q}_{0}$, then we obtain a disintegration of $\mathfrak{T}$ into type I factors in the sense of $\S 2$.

We now want to compare our results with those in Halpern [4] and Strătilă and Zsidó [18], respectively. In contrast to our approach, in these papers the decomposition of a semifinite von Neumann algebra $\Re$ into factors has been obtained on the spectrum $W$ of the center of $\Re$ with respect to a Radon measure. All the von Neumann algebras $\mathscr{T}_{\xi}, \xi \in W$, which occur in these decompositions are generated by homomorphic images of $\mathfrak{N}$. We shall now show that this implies that an analogue of Theorem 3.1 cannot be proved in the setting of these papers. In order to obtain a contradiction, we assume that an analogue of Theorem 3.1 holds. Furthermore, we assume $\mathscr{T}$ is $\sigma$-finite. It is quite easy to see that $\mathscr{N}_{\xi}$ is $\sigma$-finite too a.e. In addition, we assume $\Re$ is properly infinite and the center of $\Re$ has no atoms. Since every $\Re_{\xi}$ is generated by some homomorphic image of $\mathscr{X}$, it follows from Takesaki [23], Theorem 7, that $\mathscr{X}_{\xi}$ is the image of some normal homomorphism of $\mathscr{N}$ a.e. As $\Re_{\xi}$ is a factor a.e. we obtain from this that there exist nontrivial minimal projections in the center of $\mathcal{K}$. Thus we have reached a contradiction.

Our observations give rise to the following question. It is possible to choose the standard representation of $\Re$ and the $C^{*}$-algebra $\mathscr{Q}$ in such a manner that $\partial K(\Theta, \sigma)$ is compact?

Finally, let us consider the case of type III von Neumann algebras. For this we return to the general situation considered in $\$ 2$. We shall use the notation introduced at the beginning of $\$ 2$. Then the following can be shown.

\subsection{THEOREM. If $\mathfrak{K}_{\psi}$ is of type III, then $\mathfrak{N}_{\phi}$ is of type III a.e.}

As the proof of this theorem runs parallel with the proof of Theorem 1.1 in $\$ 4$ of Riedel [15], we give a short indication only.

Based on results of Halpern [5], Takesaki's duality theory for type III von Neumann algebras (cf. Takesaki [25]) and Proposition 2.3, a von Neumann algebra $\mathscr{B}$ can be constructed which is countably generated over its center, and a field $\left\{\Re_{\phi}\right\}_{\phi \in \Omega}$ of countably generated von Neumann algebras satisfying

$$
\mathscr{B}=\int^{\oplus} \mathscr{B}_{\phi} d \nu(\phi) \quad \text { (in the sense of Dixmier [2]) }
$$


can be found such that the following holds: If $\Re$ is of type III then $\mathscr{B}$ is of type III, and if $\mathscr{M}_{\phi}$ is of type III then $\Re_{\phi}$ is of type III. Thus the proof can be reduced to an application of the results in Lance [8].

\section{REFERENCES}

[1] E. M. Alfsen, Compact Convex Sets and Boundary Integrals, Springer-Verlag, 1971.

[2] J. Dixmier, Les algèbres d'opérateurs dans l'espace Hilbertien, Gauthier-Villars, Paris 1969.

[3] G. Emch, H. Knops and E. Verboven, Breaking of Euclidean symmetry with an application to the theory of crystallization, J. Math. Phys., 11, 5 (1970), 1655-1688.

[4] H. Halpern, Integral decompositions of functionals on $C^{*}$-algebras, Trans. Amer. Math. Soc., 168 (1972), 371-385.

[5] Normal expectations and integral decomposition of type III von Neumann algebras, Pacific J. Math., 78 (1978), 291-331.

[6] J.-P. Jurzak, Poids strictement sémi-finis et groupe d'automorphismes modulaire sur une algèbre de von Neumann décomposable, CR Acad. Sci. Paris, 279 (1974), 221-224.

[7] Decomposable operators. Applications to KMS weights in a decomposable von Neumann algebra, Rep. Math. Phys., 8 (1975), 203-228.

[8] C. Lance, Direct integrals of left Hilbert algebras, Math. Ann., 216 (1975), 11-28.

[9] J. von Neumann, On Rings of Operators. Reduction Theory, Ann. Math., 50 (1949), 401-485.

[10] G. K. Pedersen, C*-Algebras and their Automorphism Groups, Academic Press 1979.

[11] G. K. Pedersen and M. Takesaki, The Radon-Nikodym theorem for von Neumann algebras, Acta Math., 130 (1973), 53-88.

[12] N. Riedel, Über die Zerlegung von Operatoren in topologischen direkten Integralen von Hilberträumen, Manuscript Math., 23 (1978), 185-211.

[13] _ Direkte Integrale von Hilbertalgebren, Math. Ann., 246 (1980), 167-192.

[14] On reduction of tracial states, Rev. Roum. Math. Pures et Appl., 26, 6 (1981), 889-897.

[15] _ Topological direct integrals of left Hilbert algebras. I, J. Operator Theory, 5 (1981), 29-45.

[16] Topological direct integrals of left Hilbert algebras. II, J. Operator Theory, 5 (1981), 213-229.

[17] S. Sakai, $C^{*}$-algebras and $W^{*}$-algebras, Springer-Verlag, 1971.

[18] S. Strătilă and L. Szidó, An algebraic reduction theory for $W^{*}$-algebras. I, J. Functional Anal., 11 (1972), 295-313.

[19] An algebraic reduction theory for $W^{*}$-algebras. II, Rev. Roum. Math. Pures et Appl., 18 (1973), 407-460.

[20] Lectures on von Neumann Algebras, Abacus Press 1979.

[21] C. Sutherland, Direct integral theory for weights and the Plancherel formula, Bull. Amer. Math. Soc., 80 (1974), 456-461.

[22] Crossed products, direct integrals and Connes' classification of type III factors, Math. Scand., 40 (1977), 209-214.

[23] M. Takesaki, On the conjugate space of operator algebras, Tohôku Math. J., 10 (1958), 194-203.

[24] _ Tomita's Theory of Modular Hilbert Algebras and its Applications, Lecture Notes in Math., No. 128, Springer-Verlag 1970. 
[25] _ States and Automorphisms of Operator Algebras. Standard Representations and the Kubo-Martin-Schwinger Boundary Condition, in Lecture Notes in Physics, No. 20, Springer-Verlag 1973.

[26] _ Duality for crossed products and the structure of von Neumann algebras of type III, Acta Math., 131 (1973), 249-310.

[27] _ Theory of Operator Algebras. I, Springer-Verlag 1979.

[28] M. Takesaki and M. Winnink, Local normality in quantum statistical mechanics, Comm. Math. Phys., 30 (1973), 129-152.

[29] S. Teleman, Sur la réduction des algèbres de von Neumann, Rev. Roum. Math. Pures et Appl., 20 (1975), 693-742.

[30] __ On reduction theory, Rev. Roum. Math. Pures et Appl., 21 (1976), 465-486.

[31] A. van Daele, Continuous crossed products and type III von Neumann algebras, London Math. Soc., Lecture Notes Series 31, 1978.

[32] W. Wils, Direct integrals of Hilbert spaces. I, Math. Scand., 26 (1970), 73-88.

Received June 4, 1982 and in revised form April 20, 1983.

UNIVERSITY OF CALIFORNIA

BERKELEY, CA 94720 



\title{
PACIFIC JOURNAL OF MATHEMATICS EDITORS
}

\author{
Donald BABBITT (Managing Editor) \\ University of California \\ Los Angeles, CA 90024 \\ Hugo RossI \\ University of Utah \\ Salt Lake City, UT 84112 \\ C. C. MOore and Arthur Ogus \\ University of California \\ Berkeley, CA 94720
}

J. DUGUNDJI
Department of Mathematics

University of Southern California

Los Angeles, CA 90089-1113

R. FinN and H. SAMELSON

Stanford University

Stanford, CA 94305

\section{ASSOCIATE EDITORS}
R. ARENS
E. F. BECKENBACH
B. H. NeumanN
F. WOLF
K. YosHIDA (1906-1982)

\section{SUPPORTING INSTITUTIONS}

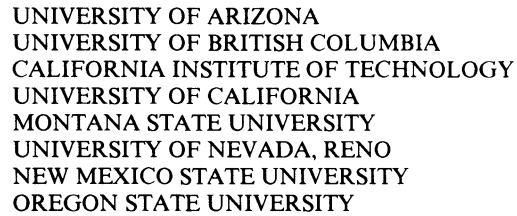

UNIVERSITY OF ARIZONA

UNIVERSITY OF BRITISH COLUMBIA

CALIFORNIA INSTITUTE OF TECHNOLOGY

UNIVERSITY OF CALIFORNIA

MONTANA STATE UNIVERSITY

UNIVERSITY OF NEVADA, RENO

NEW MEXICO STATE UNIVERSITY

OREGON STATE UNIVERSITY

\author{
UNIVERSITY OF OREGON \\ UNIVERSITY OF SOUTHERN CALIFORNIA \\ STANFORD UNIVERSITY \\ UNIVERSITY OF HAWAII \\ UNIVERSITY OF TOKYO \\ UNIVERSITY OF UTAH \\ WASHINGTON STATE UNIVERSITY \\ UNIVERSITY OF WASHINGTON
}

The Supporting Institutions listed above contribute to the cost of publication of this Journal, but they are not owners or publishers and have no responsibility for its content or policies.

Mathematical papers intended for publication in the Pacific Journal of Mathematics should be in typed form or offset-reproduced (not dittoed), double spaced with large margins. Please do not use built up fractions in the text of the manuscript. However, you may use them in the displayed equations. Underline Greek letters in red, German in green, and script in blue. The first paragraph must be capable of being used separately as a synopsis of the entire paper. In particular it should contain no bibliographic references. Please propose a heading for the odd numbered pages of less than 35 characters. Manuscripts, in triplicate, may be sent to any one of the editors. Please classify according to the scheme of Math. Reviews, Index to Vol. 39. Supply name and address of author to whom proofs should be sent. All other communications should be addressed to the managing editor, or Elaine Barth, University of California, Los Angeles, California 90024.

There are page-charges associated with articles appearing in the Pacific Journal of Mathematics. These charges are expected to be paid by the author's University, Government Agency or Company. If the author or authors do not have access to such Institutional support these charges are waived. Single authors will receive 50 free reprints; joint authors will receive a total of 100 free reprints. Additional copies may be obtained at cost in multiples of 50 .

The Pacific Journal of Mathematics is issued monthly as of January 1966. Regular subscription rate: $\$ 132.00$ a year (6 Vol., 12 issues). Special rate: $\$ 66.00$ a year to individual members of supporting institutions.

Subscriptions, orders for numbers issued in the last three calendar years, and changes of address should be sent to Pacific Journal of Mathematics, P.O. Box 969, Carmel Valley, CA 93924, U.S.A. Old back numbers obtainable from Kraus Periodicals Co., Route 100, Millwood, NY 10546.

The Pacific Journal of Mathematics ISSN 0030-8730 is published monthly by the Pacific Journal of Mathematics at P.O. Box 969, Carmel Valley, CA 93924. Application to mail at Second-class postage rates is pending at Carmel Valley, California, and additional mailing offices. Postmaster: Send address changes to Pacific Journal of Mathematics, P. O. Box 969, Carmel Valley, CA 93924.

PUBLISHED BY PACIFIC JOURNAL OF MATHEMATICS, A NON-PROFIT CORPORATION 


\section{Pacific Journal of Mathematics}

\section{Vol. 111, No. $2 \quad$ December, 1984}

Berndt Brenken, Representations and automorphisms of the irrational

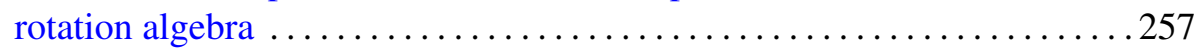

Harold George Diamond, A number theoretic series of I. Kasara . . . . . . 283

Rolf Farnsteiner, On the structure of simple-semiabelian Lie algebras . . . . 287

Guillermo Grabinsky, Poisson process over $\sigma$-finite Markov chains . ......301

Derbiau Frank Hsu and A. Donald Keedwell, Generalized complete

mappings, neofields, sequenceable groups and block designs. I . . . . . 317

William H. Julian and Fred Richman, A uniformly continuous function on

$[0,1]$ that is everywhere different from its infimum $\ldots \ldots \ldots \ldots . \ldots 33$

D. H. Lehmer and Emma Lehmer, The sextic period polynomial .........341

E. Maluta, Uniformly normal structure and related coefficients ...........357

Coy Lewis May, The species of bordered Klein surfaces with maximal

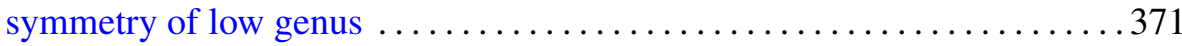

Louis Jackson Ratliff, Jr., On asymptotic prime divisors . . . . . . . . . . . 395

Norbert Riedel, Disintegration of KMS-states and reduction of standard von

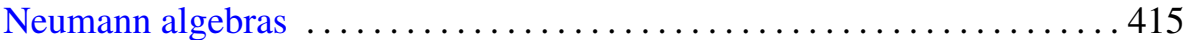

Richard Gordon Swan, $n$-generator ideals in Prüfer domains ...........433

Vilmos Totik, An interpolation theorem and its applications to positive

operators .................................. 447

Richard Vrem, Hypergroup joins and their dual objects 